Bio - grafía. Escritos sobre la Biología y su Enseñanza. ISSN 2027-1034

Edición Extraordinaria. p.p. 720 - 727

Memorias del IX Encuentro Nacional de Experiencias en Enseñanza de la Biología y la

Educación Ambiental. IV Congreso Nacional de Investigación en Enseñanza de la Biología.

\title{
EL TRABAJO DE CAMPO CON Mesamphiagrion laterale (ODONATA: COENAGRIONIDAE) COMO ESTRATEGIA EDUCATIVA PAR A LA ENSEÑ ANZA DE ECOLOGÍA DE POBLACIONES EN EL HUMEDAL LA CONEJERA BOGOTA D.C.
}

\author{
Lynda Stefany Cárdenas Guerrero
}

\section{RESUMEN}

El siguiente artículo está enmarcado dentro de la investigación realizada como trabajo de grado de la Licenciatura en Biología en la Universidad Pedagógica Nacional, mostrando aquí los principales resultados de dicho proyecto el cual tiene como objetivo principal diseñar una estrategia educativa para la enseñanza y aprendizaje de la ecología de poblaciones a partir de estudios en campo con Mesamphiagrion laterale el Humedal La Conejera, Bogotá D.C.. Para esto se realizó la investigación de tres atributos poblacionales: tamaño poblacional, distribución sexual y distribución espacial de $M$. laterale un odonato encontrado en el Humedal La Conejera, en este artículo se presentan los dos primeros atributos; metodológicamente se establecieron 10 zonas de muestreo en diferentes ubicaciones del humedal teniendo 4 visitas por parcela, se empleó el método captura- recaptura. Posteriormente para la fase educativa, se desarrollan 10 sesiones de clase en el Colegio Tibabuyes Universal con 40 estudiantes de grado noveno para diseñar la estrategia educativa en torno a la ecología poblacional a partir del trabajo y experiencia biológica en campo; se presentan 5 elementos educativos producto del encuentro con los estudiantes: Ideas previas, abordaje conceptual de la ecología de poblaciones, establecimiento de metodologías para el estudio de poblaciones, experiencias prácticas y proceso de organización y sistematización de datos.

PALABRAS CLAVE: Población, Elementos Educativos, Humedal La Conejera, Captura Re-Captura.

\section{ABSTRACT}

The Following article is frame into a developed research as grade work of Biology teacher of Pedagógica Nacional University, to show there the main results of project, has a purpose to design a educative strategy to teaching and learning of population ecology from the field research with Mesamphiagrion laterale in the Conejera Wetland in Bogota D.C.

Therefore to realice a research of three population attributes: Population size, sexual distribution and spacial distribution of Odonato $M$. laterale found in the Conejera Wetland,

\footnotetext{
${ }^{1}$ Licenciada en Biología, Universidad Pedagógica Nacional, línea de investigación: Enseñanza de la ecología, Grupo de investigación: CASCADA, correo: lindasa95@hotmail.com.
} 
Bio - grafía. Escritos sobre la Biología y su Enseñanza. ISSN 2027-1034

Edición Extraordinaria. p.p. 720 - 727

Memorias del IX Encuentro Nacional de Experiencias en Enseñanza de la Biología y la

Educación Ambiental. IV Congreso Nacional de Investigación en Enseñanza de la Biología.

in this article the first and second attribute are present; the metodological procediments are to establish 10 areas to sampling in a different location of Wetland, and visiting in a 4 moments, the capture-recapture method was used. Similary for the education part, has development 10 class sessions in the Tibabuyes Universal school with $4^{\circ}$ students of $9^{\circ}$ to design a educative estrategy to learn population ecology from the work and biological experience of Wetland. The Project propose 5 educational estrategy result of students and teacher research: Previous ideas, conceptual approach of population ecology, establishment of methodologies for working with populations, practical experiences and process of organization and systematization of population data.

KEYWORDS: Population, educational element, Conejera Wetland, Capture- recapture.

\section{INTRODUCCIÓN}

En la enseñanza de la Biología, el maestro debe apostar a la realización de proyectos de investigación que favorezcan y profundicen conocimientos específicos, para dar lugar a espacios de formación investigativa con sus estudiantes; ahora bien, se encuentra que una rama importante de la Biología son las poblaciones, que permiten ampliar la visión de lo vivo holística y sistemáticamente al comprender dinámicas del medio natural a partir del estudio ecológico de la influencia del medio sobre las mismas.

Con esto, si el maestro opta por el desarrollo de proyectos de investigación escolar en ecología de poblaciones, deberá establecer criterios a modo de elementos educativos que fundamenten su enseñanza y favorecen el aprendizaje conjunto (estudiante/maestro), entrelazando así fronteras entre ciencia y escuela para orientar a los estudiantes en la construcción más que conceptos, de experiencias de vida y de reconocimiento de espacios de biodiversidad cercana.

\section{METODOLOGIA}

El presente proyecto tiene como enfoque la investigación Cualitativa- Interpretativa en donde a partir de acercamientos al contexto de los sujetos, se establecen escenarios de investigación en relación a una temática que en este caso es ecología de poblaciones, teniendo como referente el trabajo en campo con las libélulas.

De esta manera para este proyecto de investigación se toma un enfoque de investigación mixto desde Pereira (2011). Por otro lado se retoma como método la investigación como estrategia pedagógica (IEP) que según (García, 2015) intenta acercar al estudiante a situaciones un poco semejantes a la de los científicos pero desde su contexto, dándole al educando un papel activo, valorando sus conocimientos previos, permitiéndole plantear su propia postura frente a la información que no sólo está abordando, sino también construyendo, desde el desarrollo de procesos investigativos. 
Bio - grafía. Escritos sobre la Biología y su Enseñanza. ISSN 2027-1034

Edición Extraordinaria. p.p. 720 - 727

Memorias del IX Encuentro Nacional de Experiencias en Enseñanza de la Biología y la

Educación Ambiental. IV Congreso Nacional de Investigación en Enseñanza de la Biología.

Para dar un orden al proyecto se establecen las siguientes etapas que dan respuesta a los objetivos específicos:

FASE 1: Tamaño poblacional y distribución sexual de $M$. laterale en la zona de recorrido y de conservación del Humedal La Conejera

Se estableció un área de $200 \mathrm{~m}^{2}$ dividida en 10 parcelas de $20 \mathrm{~m} 2$, se llevaron a cabo 4 visitas por zona con espacio 8-10 días entre agosto- septiembre de 2016; se realizaron 3 eventos de captura- recaptura (marcaje) y se implementaron fichas de descripción, determinación de sexo y uso de geoposicionador.

FASE 2: Diseño y desarrollo de la estrategia educativa con la comunidad en relación a tres atributos de la población de libélulas:

Se desarrollaron 10 sesiones de clase (7 teórico-prácticas, 3 prácticas de campo); los temas de las clases abordan: Ideas previas, abordaje conceptual de insectos, simulacro de cuaderno de campo en huerta, abordaje conceptual de atributos poblacionales, distribución espacial en el colegio, simulacro de prácticas de campo y 3 salidas de campo al Humedal La Conejera.

\section{RESULTADOS Y DISCUSIÓN}

\section{PRIMERA FASE :}

Para escoger la especie de insecto a trabajar se tuvo en cuenta lo siguiente: Que se encuentre en zonas accesibles al humedal, que sea considerada inofensiva, de fácil acceso y estudio para el profesor y estudiantes, que sea una especie cosmopolita y abundante en el humedal, es decir que tenga una presencia significativa; escogiendo de esta manera a Mesamphiagrion laterale para el trabajo biológico y con los estudiantes.

De acuerdo al tiempo de estudio correspondiente a la investigación en campo, se capturaron, marcaron y liberaron 1352 individuos de Mesamphiagrion laterale en los tres estadíos de crecimiento (tenerales, juveniles adultos) de los cuáles se tuvo un porcentaje de recaptura de $10.06 \%$ correspondiente a 136 individuos en total por las 10 zonas.

El mayor tamaño poblacional promedio fue en la zona 4 con 3836,279 individuos y la zona 3 con 2076,444 individuos, lo que puede deberse principalmente a que fueron las únicas parcelas junto a cuerpos de agua, lo que según (Angelibert \& Giani 2003) es relevante ya que los odonatos inician su ciclo de vida en cuerpos de agua y los adultos se alimentan de otros insectos que también tienen ciclo de vida similar o simplemente posan en las riberas (Corbet, 1962); también se encuentra baja proporción de recapturas en las parcelas, en este caso se excluye la manipulación de los especímenes como causa, ya que este método de marcaje de alas con color rojo fue probado para esta especie y 
Bio - grafía. Escritos sobre la Biología y su Enseñanza. ISSN 2027-1034

Edición Extraordinaria. p.p. 720 - 727

Memorias del IX Encuentro Nacional de Experiencias en Enseñanza de la Biología y la

Educación Ambiental. IV Congreso Nacional de Investigación en Enseñanza de la Biología.

hábitat similar corroborando que no hay repercusiones en las recapturas o supervivencia (Palacino y Contreras, 2014).

Con respecto a la distribución sexual, se encuentra un sesgo general hacia machos, teniendo zonas con proporción 3:1, por ejemplo en la primera zona de conservación se encuentra una proporción 250 Machos/ 54 Hembras, esta situación está bien documentada según (Cordero y Stoks, 2008) teniendo como posibles caus as un sesgo en la emergencia del adulto o emergencia asincrónica, también puede deberse a las visitas esporádicas de las hembras a cuerpos de agua, información que se corrobora al encontrar inclinación hacia los machos en parcelas cercanas a cuerpos de agua (zona 1 y 2 de conservación, zona 3 y 4 del sendero) igualmente se corrobora al tener resultados contrarios, es decir un sesgo hacia hembras en parcelas lejanas a cuerpos de agua (zona 6,7 y 8), lo que se debe principalmente a la maduración de huevos lejos de cuerpos de agua según (Corbet, 1952).

Estos hallazgos concuerdan con el estudio de Pulido (2014) en donde para M. laterale en el Humedal Córdoba solo se encuentran dos hembras en el total de las capturas, igualmente Palacino y Contreras (2014) reportan a esta especie en zonas de Guatavita en proporción 3:1

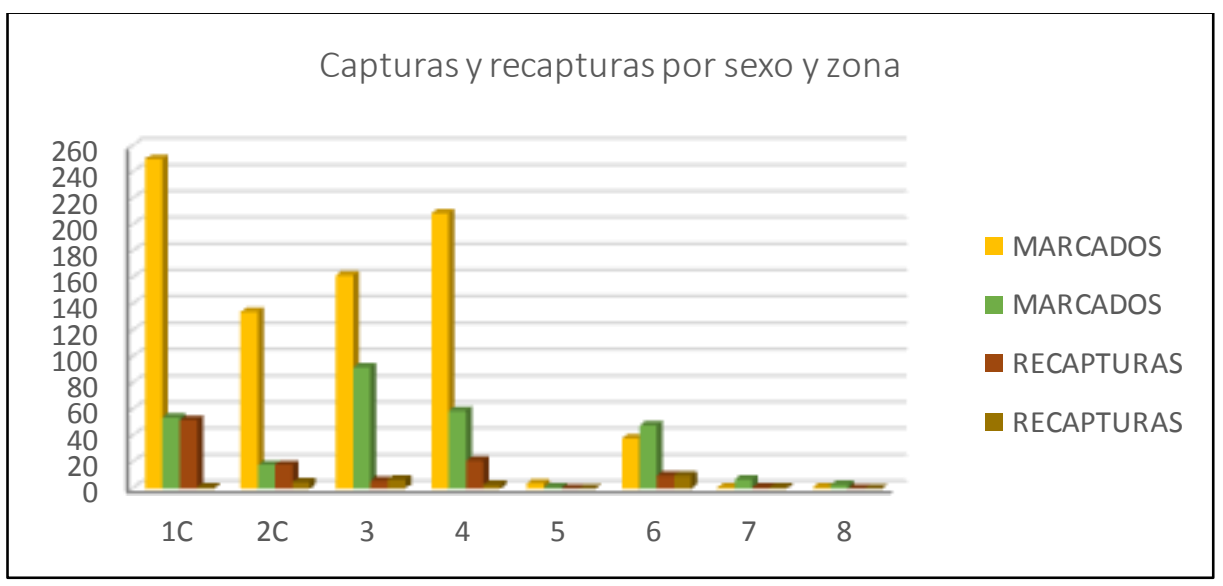

Ilustración 1: Proporción de capturas y recapturas (se discriminan los datos de la zona 1 y 2 al no encontrar capturas)

\section{SEGUNDAFASE:}

Para llevar a cabo el segundo objetivo específico, el cual pretende determinar los elementos educativos para la enseñanza de la ecología de poblaciones involucrando el trabajo biológico de campo, se establecen los siguientes elementos educativos:

1. Reconocimiento de ideas previas: 


\section{Bio - grafía. Escritos sobre la Biología y su Enseñanza. ISSN 2027-1034}

\section{Edición Extraordinaria. p.p. 720 - 727}

Memorias del IX Encuentro Nacional de Experiencias en Enseñanza de la Biología y la

Educación Ambiental. IV Congreso Nacional de Investigación en Enseñanza de la Biología.

Comprende la sesión 1 y 2 en donde se identificaron las principales características de la clase Insecta y se trabajó con láminas de chocolatina Jet para identificar ideas sobre población, individuo, comunidad, ecosistema, encontrando respuestas como:

"Es una población porque se identifican al tener las mismas características y por eso es una población, porque es un conjunto de la misma especie, estas características son: terrestres" UI \#1

Se identifica que las ideas previas de los estudiantes para población, se encuentran inclinadas hacia el agrupamiento o unión de individuos de la misma especie, siendo la definición de especie dada por semejanzas en las características morfológicas 0 comportamentales; igualmente los estudiantes no involucran tiempo y rara vez espacio, encontrando que para ellos una población puede estar en varias zonas al mismo tiempo, según (Jiménez, 2009) "la variable tiempo es escasa, y se tiene en cuenta asociada a ejemplos muy concretos, lo que supone un obstáculo para comprender procesos asociados a la dinámica de las poblaciones, siendo mayor para periodos largos de tiempo".

\section{Abordaje conceptual de la ecología de poblaciones:}

Fueron abordadas temáticas centrales a partir de un mapeo de conceptos en ecología de poblaciones en las sesiones 2,4 y 5 , se reconoció familiaridad en los estudiantes con los conceptos de natalidad, mortalidad y migración principalmente, sin embargo se encuentra que la aplicación de los mismos en las poblaciones naturales se vuelve confusa y enredada, en donde fue frecuente la confusión entre atributos de los individuos y de las poblaciones, encontrando respuestas como:

\section{"Hay aves que no viven en población" Ul\#2}

\section{"Son diferentes especies cuando cambian de lugar" Ul\#3}

En la enseñanza y aprendizaje de las ciencias es fundamental dar lugar a la construcción de un conocimiento teórico acompañado con el conocimiento estudiantil, que de sentido a la razón del porque aprender sobre ciertos temas, en este sentido (Quintanilla, 2006) reconoce que "El estudiante puede no conocer la finalidad de lo que aprende y es entonces cuando la ciencia en la escuela pierde significado, sentido y valor. Por todo esto, se hace necesaria una teoría de los contenidos científicos escolares, que surja de un modelo general de ciencia" (p.187).

\section{Preparación y establecimiento de metodologías para el trabajo con poblaciones}

Se lleva a cabo la sesión 3 y 7 la cual por medio de grupos de trabajo, se realizó un simulacro de prácticas de campo, reconociendo distribución espacial, distribución sexual y tamaño poblacional con especímenes preservados de $M$. laterale, en donde por grupos de trabajo, se entregó protocolos de campo para el trabajo con la población de acuerdo al atributo escogido.

En este punto se resalta que los estudiantes tuvieron procesos de organización en cuanto a la presentación de los datos, encontrando por ejemplo convenciones como $\mathrm{H}$ para 
Bio - grafía. Escritos sobre la Biología y su Enseñanza. ISSN 2027-1034

Edición Extraordinaria. p.p. 720 - 727

Memorias del IX Encuentro Nacional de Experiencias en Enseñanza de la Biología y la

Educación Ambiental. IV Congreso Nacional de Investigación en Enseñanza de la Biología.

designar a hembras y $\mathrm{M}$ para machos tanto en las gráficas como en las tablas; se encontró que para muchos estudiantes, esta actividad fue el primer acercamiento a la manipulación con especímenes, lo anterior fortalece la investigación escolar como proceso de mucha importancia en la comprensión no solo de las ciencias, sino de situaciones que hacen parte del contexto cercano, en relación a esto (Cano, 2009) menciona que "las situaciones de aprendizaje a partir de las que el alumnado construye sus conocimientos sobre lo que es una investigación han de ser necesariamente de investigación" (p.74).

\section{Experiencias prácticas}

Comprende las sesiones 3, 6, 7, 8, 9 y 10 determina las prácticas sobre ecología de poblaciones ya sea en el salón de clases, la huerta escolar o el Humedal La Conejera; en esta parte se evidencia mayor profundidad en las descripciones del cuaderno de campo de los estudiantes:

"Macho: con rayas azules en el tórax y alas cuadradas, Hembra: dos semanas de haber salido del agua, pigmentación pálida, Hembra: casi adulta y tiene el abdomen casi negro, (sin determinar) UI \#4

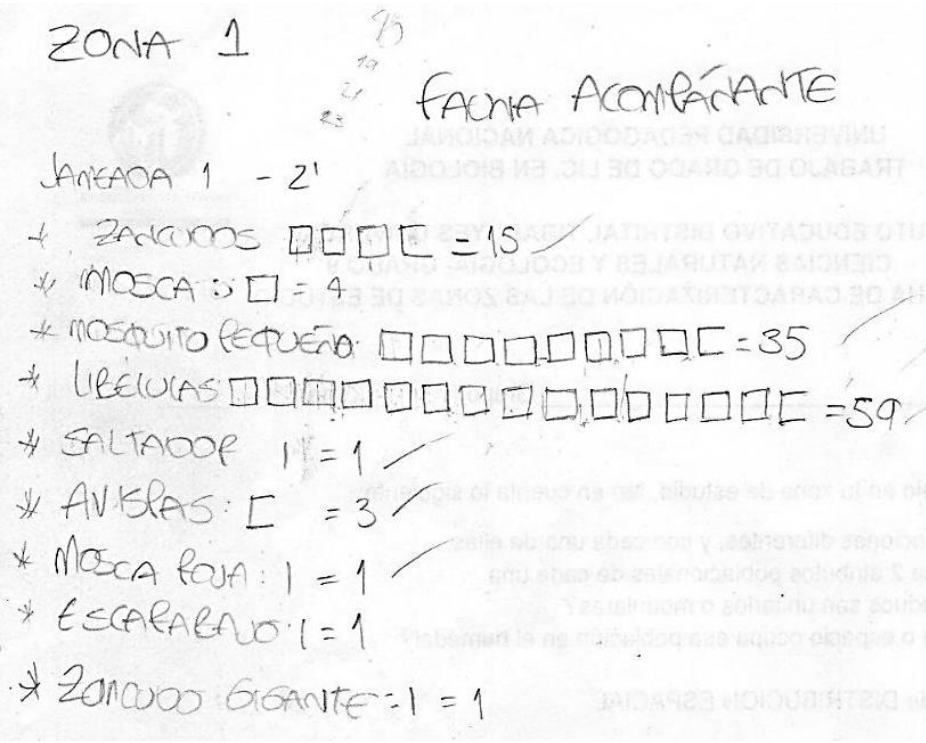

Ilustración 2: Sistematización de fauna acompañante. Autoría de los

Se encuentran resultados similares al trabajo biológico en campo del maestro: estudiantes

"Hay más libélulas en la fase adulta, porque hay más machos adultos que hembras". UI \#5

Es así como se encuentra una correlación entre la investigación escolar y la investigación científica, resaltando lo que menciona (Barberá y Valdés, 1996) "debemos ser conscientes de que los alumnos sólo aprenderán a hacer ciencia practicándola -al igual que ocurre con los científicos- siguiendo sus líneas propias de indagación, lo que les permitirá conocer que hacer ciencia no es fuertemente dependiente de la teoría, sino también de la práctica" (p.374).

\section{Manejo de información}

Dado por todos aquellos procesos o habilidades que desarrollan los estudiantes con la información dada por el maestro además de lo que se evidencia en el ecosistema y unido a lo que ellos conocen, de esta manera este elemento estuvo predominado por las prácticas de campo de las sesiones 8,9 y 10, en donde se reconoce la recopilación de datos de manera adecuada y propositiva ya que los estudiantes hicieron fichas propias de 
Bio - grafía. Escritos sobre la Biología y su Enseñanza. ISSN 2027-1034

Edición Extraordinaria. p.p. 720 - 727

Memorias del IX Encuentro Nacional de Experiencias en Enseñanza de la Biología y la

Educación Ambiental. IV Congreso Nacional de Investigación en Enseñanza de la Biología.

clasificación de la fauna acompañante que se encontraba en la colecta de los odonatos (llustración 2)

lgualmente se evidencian procesos de inferencia sobre los fenómenos biológicos observados, es decir el uso de terminología científica en las descripciones y fichas de observación:

"Por el hecho de que los mosquitos les sirve de alimentación a las libélulas se encontraron especies diferentes" UI \#6

Se puede señalar, que la visión de los estudiantes estuvo encaminada en el entendimiento del tamaño poblacional a partir de la disponibilidad del recurso alimento, en este sentido, se encuentra una relación con lo que (Develay y Ginsburger-Vogel, 1986) señalan "Las relaciones alimentarias constituyen una buena aproximación a los problemas de fluctuaciones poblacionales donde los alumnos suelen formular más o menos hábilmente todos los criterios que intervienen (la cantidad de alimento disponible, la concurrencia interespecífica, la tasa de fecundidad)" (p.45).

\section{CONCLUSIONES}

- La proporción de sexos de M. laterale en el Humedal la Conejera presenta un sesgo general hacia los machos, el tamaño poblacional no es constante para la zona de recorrido y conservación del Humedal, estando influenciado principalmente por presencia de presas y cuerpos de agua cercanos, siendo frecuente la ubicación de parches de individuos en zonas con cobertura de pasto tipo Kikuyo.

- En la enseñanza de la ecología de poblaciones es pertinente la inclusión de metodologías específicas para el trabajo en campo y la recolección de información, dichos procedimientos deben estar encaminados en la realización de protocolos que brinden orientaciones al estudiante acerca de formas de recolectar datos y sistematizarlos pero que además posibiliten dinámicas autónomas y propositivas sobre los fenómenos que el estudiante observa en campo.

- La implementación de experiencias prácticas con los estudiantes especialmente las prácticas de campo, fueron adecuadas para la enseñanza de la ecología de poblaciones ya que permitieron un contraste entre la teoría abordada en el aula y las dinámicas de la población en campo, lo que enriqueció considerablemente el proyecto de investigación y favoreció los procesos de enseñanza y aprendizaje.

\section{BIBLIOGRÁFIA CITADA}

> Angelibert, S. \& Giani, N. (2003). Dispersal Characteristics of Three Odonate Species in a Patchy Habitat. Ecography. 26(1), 13-20. 
Bio - grafía. Escritos sobre la Biología y su Enseñanza. ISSN 2027-1034

Edición Extraordinaria. p.p. 720 - 727

Memorias del IX Encuentro Nacional de Experiencias en Enseñanza de la Biología y la Educación Ambiental. IV Congreso Nacional de Investigación en Enseñanza de la Biología.

> Altamiranda, M. y Ortega, O. (2012) Estructura poblacional de Polythore gigantea (Odonata: Polythoridae) en sistemas loticos con diferentes estados de conservación en Antioquia, Colombia. Revista de Biología tropical, 60(3), 1205-1216.

$>$ Bazán- Delgado, A. (2013). La biología y su lenguaje científico, un análisis lingüístico. Santiago, (133), 261-671.

> Bota- Sierra, C. y Wolf, M. (2013). Taxonomic revisión of Mesamphiagrion Kennedy, 1920 from Colombia (Odonata: Coenagrionidae), with the description of the four new species. Zootaxa, 3718 (5), 401-440.

$>$ Berzal De Pedrazzini, M. y Barberá, O. (1993) ideas sobre el concepto biológico de población. En Enseñanza de las ciencias. 11. 149-159.

> Corbet, P. (1962). A Biology of Dragonflies. London: H. F. \& G. Witherby LTD.

> Cordero, A. R., \& Stoks, R. (2008). Mark- recapture studies and demography. En Córdoba-Aguilar, A. (Ed.), Dragonflies and damselflies: Model Organisms for Ecological and Evolutionary Research (pp.7-20). Nueva York: Estados Unidos: Oxford.

> Develay, M. y Ginsburger-Vogel, Y. (1986). Population. Institut national de recherche pédagogique, Paris: Francia.

> Garcia, S. (2015). Metodologías didácticas para la enseñanza y aprendizaje de las ciencias naturales en zonas rurales del municipio de Obando - Valle del Cauca. (Tesis de maestría). Universidad Nacional de Colombia- sede Palmira, Valle del Cauca, Colombia.

> Jimenez, M. (2009). Los conceptos de población y especie en la enseñanza de la Biología: concepciones, dificultades y perspectivas (Tesis doctoral). Universidad de Granada. España. (p.7-44).

> Palacino, F. y Contreras, N. (2014). Does experimental marking of wings influence resighting success in Mesamphiagrion laterale and Erythrodiplax umbrata (Odonata: Coenagrionidae, Libellulidae). Odonatologica. 43(3/4), 237-246.

> Pereira, Z (2011). Los diseños de método mixto en la investigación en educación: Una experiencia concreta. Revista electrónica educare, 15 (1), 15-29.

$>$ Pulido, L. (2014). Etapas de madurez sexual identificadas por coloración en Mesamphiagrion laterale (Zygoptera: Coenagrionidae). Recuperado de: https://www.academia.edu/10355969/Etapas de madurez sexual identificadas por c oloraci\%C3\%B3n en Mesamphiagrion laterale Zygoptera Coenagrionidae.

> Sanchez, F. y Pontes, A. (2010). La comprensión de conceptos de ecología y sus implicaciones para la educación ambiental. Eureka, 7 (número extraordinario), 271-285. 